\title{
Hypothesis
}

\section{How Our Neanderthal Genes Affect the COVID-19 Mortality: Iran and Mongolia, Two Countries with the Same SARS- CoV-2 Mutation Cluster but Different Mortality Rates}

\author{
Mortazavi S. A. R. ${ }^{1 \odot}$, Kaveh-Ahangar K. ${ }^{2}$, Mortazavi S. M. \\ J. ${ }^{3}$, Firoozi D. ${ }^{4}$, Haghani M.5*०
}

\begin{abstract}
Neanderthal genes possibly gave modern human protection against viruses. However, a recent study revealed that that a long sequence of DNA that is inherited from our Neanderthal ancestors can be linked to severe COVID-19 infection and hospitalization. Substantial evidence now indicates that our genetic background may be involved in the transmissibility of SARS-CoV-2 and the rapid progress of COVID-19 in some infected individuals. Although both morbidity and mortality of COVID-19 strongly depends on key factors such as age and co-existing health conditions, potential classes of human genomic variants possibly affect the likelihood of SARS-CoV-2 infection and its progress. Despite Iran and Mongolia seem to share the same SARS-CoV-2 mutation cluster, the COVID-19 mortality rates in these two countries are drastically different. While the population in Iran is 25.8 times higher than that of Mongolia, the number of confirmed cases is 1170 times higher. Moreover, the death rate shows a drastic difference. Since Neanderthals interbred with modern humans in Middle East between 47,000 and 65,000 years ago before going extinct 40,000 years ago, some Iranians have much more Neanderthal DNA than other people. Although neither genetic background nor environmental factors alone can determine our risk of developing severe COVID-19, our genes clearly affect both the development and progression of infectious diseases including COVID-19. Given these considerations, we believe that these great differences, at least to some extent, can be due to the proportion of Neanderthal genes among the people of these two countries.
\end{abstract}

Citation: Mortazavi SAR, Kaveh-Ahangar K, Mortazavi SMJ, Firoozi D, Haghani M. How Our Neanderthal Genes Affect the COVID-19 Mortality: Iran and Mongolia, Two Countries with the Same SARS-CoV-2 Mutation Cluster but Different Mortality Rates. J Biomed Phys Eng. Mortality: Iran and Mongolia, Two Countries with the Sanc

Keywords

COVID-19; SARS-Cov-2; Neanderthals; Genes; Coronavirus; Viral Diseases

\section{Introduction}

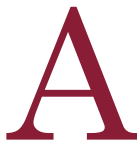

s of December 42020, SARS-CoV-2, the novel coronavirus that causes COVID-19, has killed more than 1.5 million people in different countries. The number of confirmed COVID-19 infections has surpassed 65.4 million, globally. Environmental factors can change the expression of specific genes. The genetic and molecular basis of human adaptation and evolution has been known for decades. An accumulating body of evidence now indicates that our genetic background may control the transmissibility of SARS-CoV-2. Moreover, the genetic background can explain why the progress of COVID-19 is so fast in
${ }^{1} \mathrm{MD}$, School of Medicine, Shiraz University of Medical Sciences,

Shiraz, Iran

${ }^{2} \mathrm{MSc}$, Vice-Chancellery

for Research, Shiraz

University of Medical Sci-

ences, Shiraz, Iran

${ }^{3} \mathrm{PhD}$, Department of

Medical Physics, School

of Medicine, Shiraz

University of Medical Sci-

ences, Shiraz, Iran

${ }^{4} \mathrm{PhD}$ Candidate, Depart-

ment of Clinical Nutri-

tion, School of Nutrition

and Food Sciences, Shi-

raz University of Medical

Sciences, Shiraz, Iran

${ }^{5} \mathrm{PhD}$, Department of Radiology, School of Para-

medical Sciences, Shiraz

University of Medical

Sciences, Shiraz, Iran

*Corresponding author: M. Haghani

Department of Radiol-

ogy, School of Para-

medical Sciences, Shiraz

University of Medical

Sciences, Shiraz, Iran

E-mail: m.haghani4744@

yahoo.com

Received: 31 October 2020

Accepted: 10 December 2020 
some infected individuals. Both morbidity and mortality of COVID-19 strongly depends on key factors such as age and co-existing health conditions. However, potential classes of human genomic variants can also affect the likelihood of SARS-CoV-2 infection and its progress.

\section{SARS-CoV-2 Variants around the World}

SARS-CoV-2, like other coronaviruses, has a single-stranded genomic RNA, approximately $30 \mathrm{~kb}$ in length [1]. RNA viruses mutate and evolve much faster than their hosts (e.g. up to a million times higher than their hosts such as humans). RNA viruses mutate faster than DNA viruses. These high rates of mutation in RNA viruses have been reported to be associated with enhanced virulence [2]. Moreover, when an antiviral treatment is not robust and hence unable to eliminate viruses, the exerted selective pressure endangers the fitness of virus and viral evolution is promoted. In this case, the virus has a great opportunity to evolve through adaptive mutations. This issue may play a key role in limiting the success of infection control. SARS-CoV-2 control must consider its evolutionary and adaptive mutation characteristics. Today, tens of millions of people around the world are infected with the virus. Therefore, the widespread use of non-fully effective an- tiviral agents/vaccines can trigger a global disaster. Given this consideration, developing an efficient treatment against SARS-CoV-2 is of key importance. As a 100\% selective pressurefree treatment [3], low dose radiation therapy (LDRT) has opened new horizons in the treatment of COVID-19. LDRT for COVID-19 associated pneumonia and acute respiratory distress syndrome (ARDS), was introduced by our team in March 2020 and subsequently this idea has been supported by other scientists from different countries. Using LDRT is not only based on anti-inflammatory effects of low dose radiation but also on properties including optimizing the activity of the immune system and preventing thrombosis.

Iran and Mongolia, Two Countries with Puzzling COVID-19 Mortalities

Today, there is a global race to determine which factors drive the severity of COVID-19. These efforts are focused on finding which human or viral factors determine an individual infected by SARS-CoV-2 will develop severe symptoms [4]. Although some studies show that Iran and Mongolia may share the same SARS-CoV-2 mutation cluster (Figure 1), the COVID-19 mortality rates in these two countries are drastically different (Figure 2). While the population in Iran is about 26 times higher than that of Mongolia, the number of con-

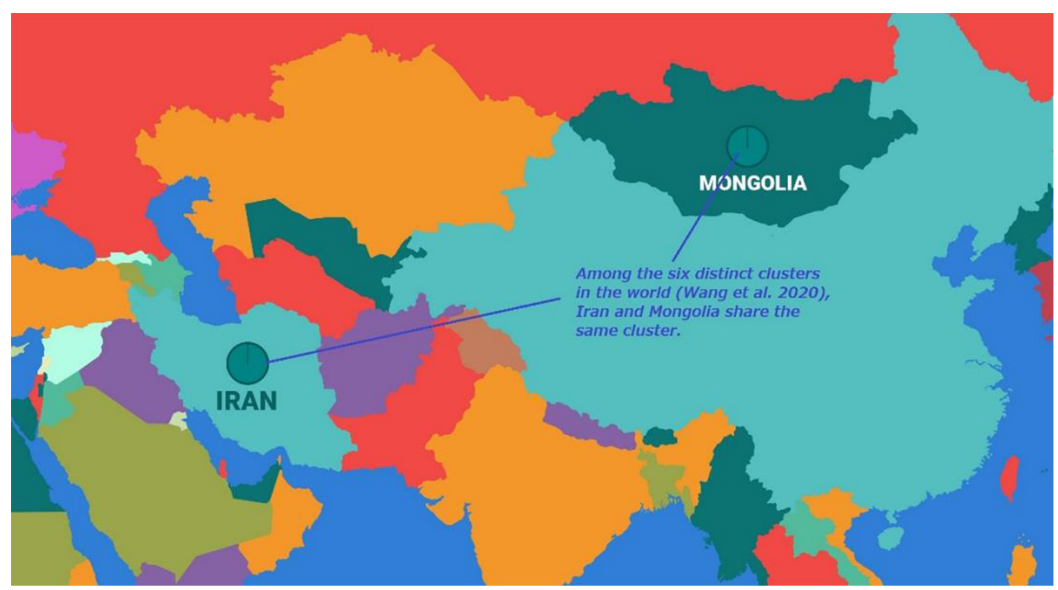

Figure 1: Iran and Mongolia share the same SARS-CoV-2 mutation cluster (Figure reproduced from Chen M, et al. J Mol Biol. 2020;432(19):5212-6. [5]) 
Neanderthal Genes and Severity of the COVID-19

firmed cases is 1170 times higher (Figure 2).

Moreover, the death rate shows a drastic difference. Hundreds of genetic variants that can be traced to the Neanderthals have been identified. According to currently accepted theories, Neanderthals interbred with modern humans in Middle East between 47,000 and 65,000 years ago before going extinct 40,000 years ago (Figure 3). Thus, it's not puzzling that some Iranians have much more Neander-

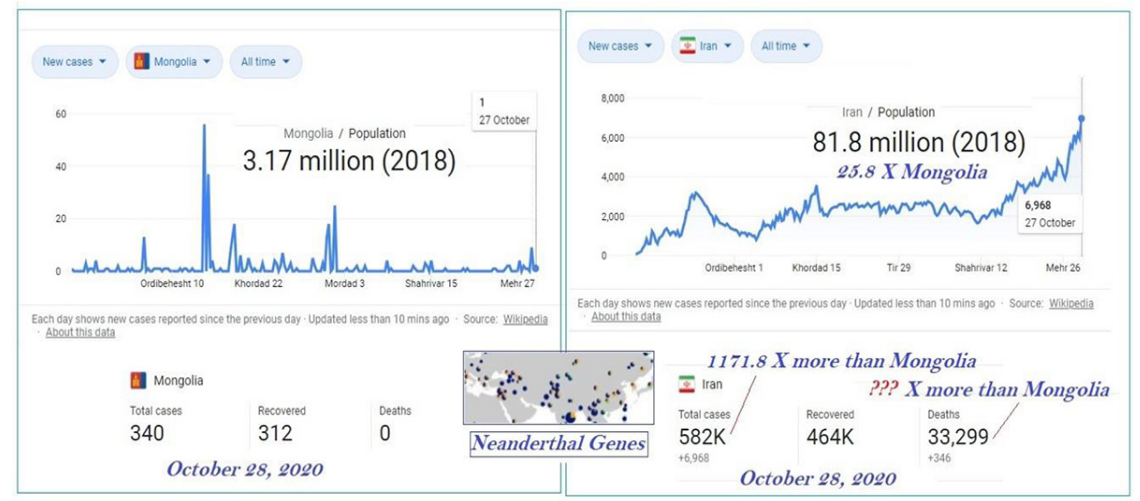

Figure 2: The COVID-19 cases and total deaths in Iran and Mongolia (Figure reproduced from data provided by Wikimedia and illustrated by Google)

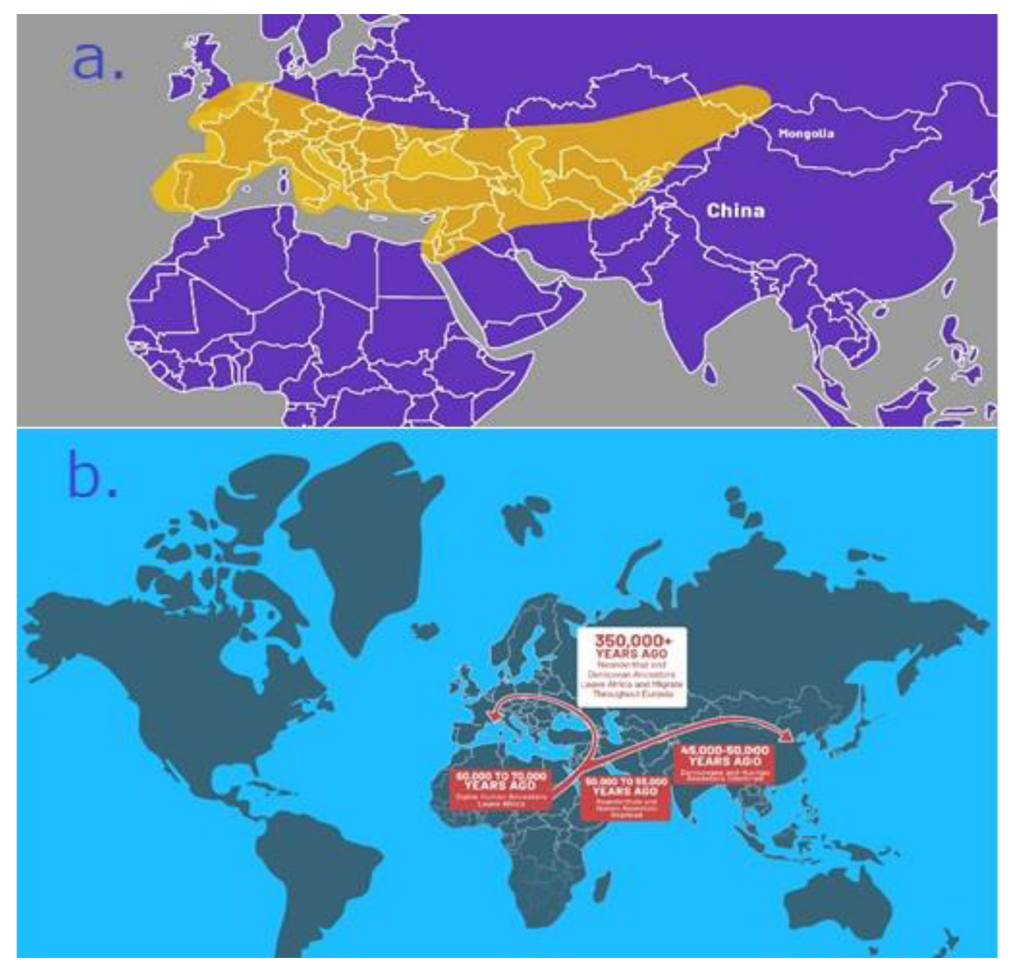

Figure 3: Neanderthals interbred with modern humans in Middle East between 47,000 and 65,000 years ago before going extinct 40,000 years ago. a. The geographical range of Homo Neanderthalenisis. b. About 60,000-70,000 years ago, the ancestors of modern human began interbreeding with Neanderthals. Estimates show that the majority of the Neanderthal DNA in the genomes of modern human is originated from interbreeding that occurred around 50,00055,000 years ago in the Middle East. (Figure reproduced from: https://www.the-scientist.com/ features/neanderthal-dna-in-modern-human-genomes-is-not-silent-66299 [6]). 
thal DNA than other people (Figure 4).

It was previously reported that Neanderthal genes possibly gave modern human protection against viruses. In 2018, Stanford scientists reported that the genomes of humans and other species contain signatures of ancient epidemics. They concluded that Inherited Neanderthal genes protect us against viruses [7]. However, a recent study revealed that a gene cluster on chromosome 3 is a risk locus for respiratory failure in severe COVID-19 patients. Zeberg and Pääbo have recently reported that a long sequence of DNA that can be linked to severe COVID-19 infection and hospitalization is inherited from our Neanderthal ancestors [8]. It is worth noting that the sequence is not evenly distributed across modern human populations [9]. Figure 5 shows the frequency at which the risk factor is found in various populations around the globe. The map shows the geographic distribution of the Neanderthal core haplotype that determines the risk for severe COVID-19. It should be noted that genetic background and environmental factors alone do not determine our risk of developing severe COVID-19. However, both the development and progression of any infectious diseases such as COVID-19 can be clearly determined by our genes [9].

In summary, Iran and Mongolia are two Asian countries that share the same SARSCoV-2 mutation cluster. However, the COVID-19 mortality rates in these two countries are drastically different. The Neanderthals interbred with modern humans in Middle East between 47,000 and 65,000 years ago before disappearing 40,000 years ago. Thus, some Iranians have much more Neanderthal DNA than people in other countries. Neanderthal genes possibly gave modern human protection against viruses. However, a recent study revealed that that a long sequence of DNA that

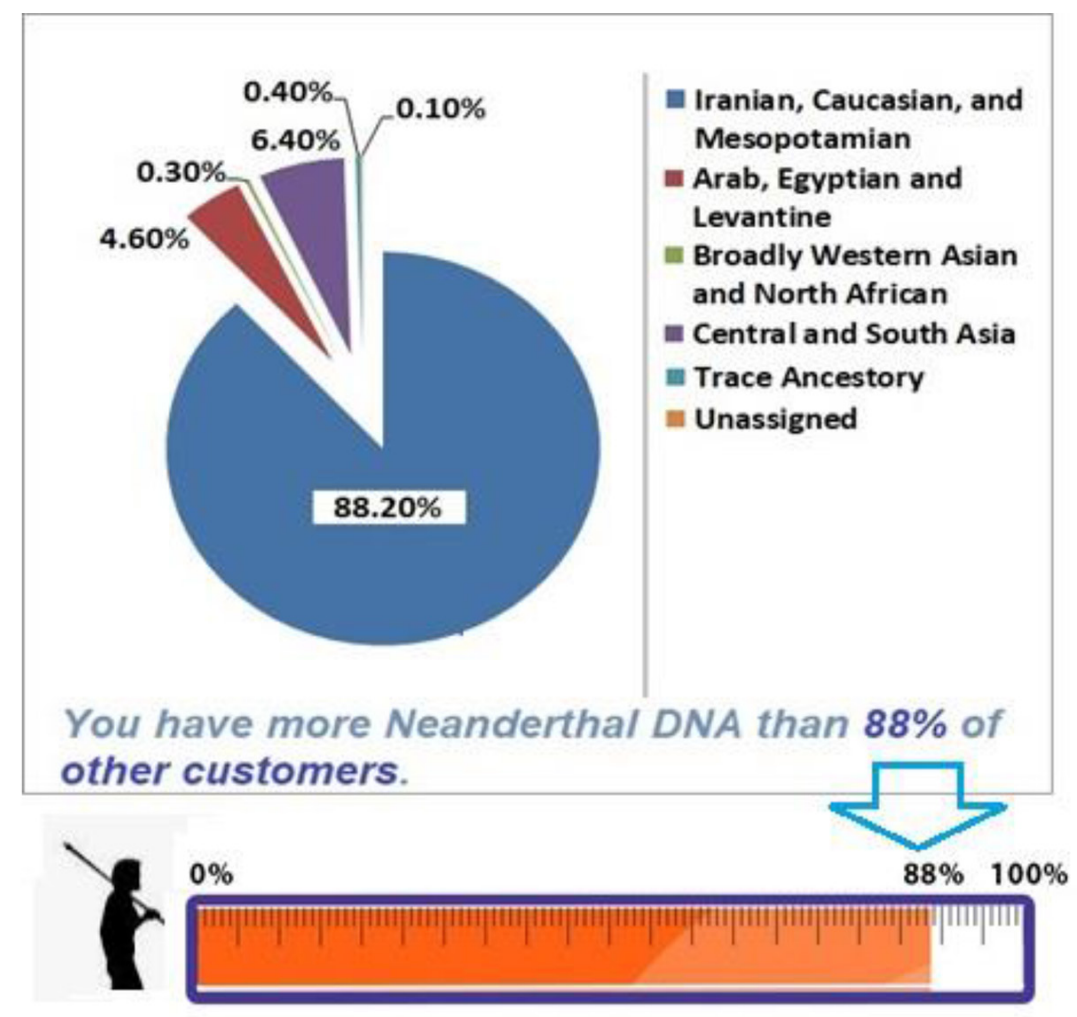

Figure 4: The genome analysis of an Iranian man that indicates he has more Neanderthal DNA than about $90 \%$ of other people tested. (Based on the results of genome analysis of an Iranian customer; www.23andme.com). 


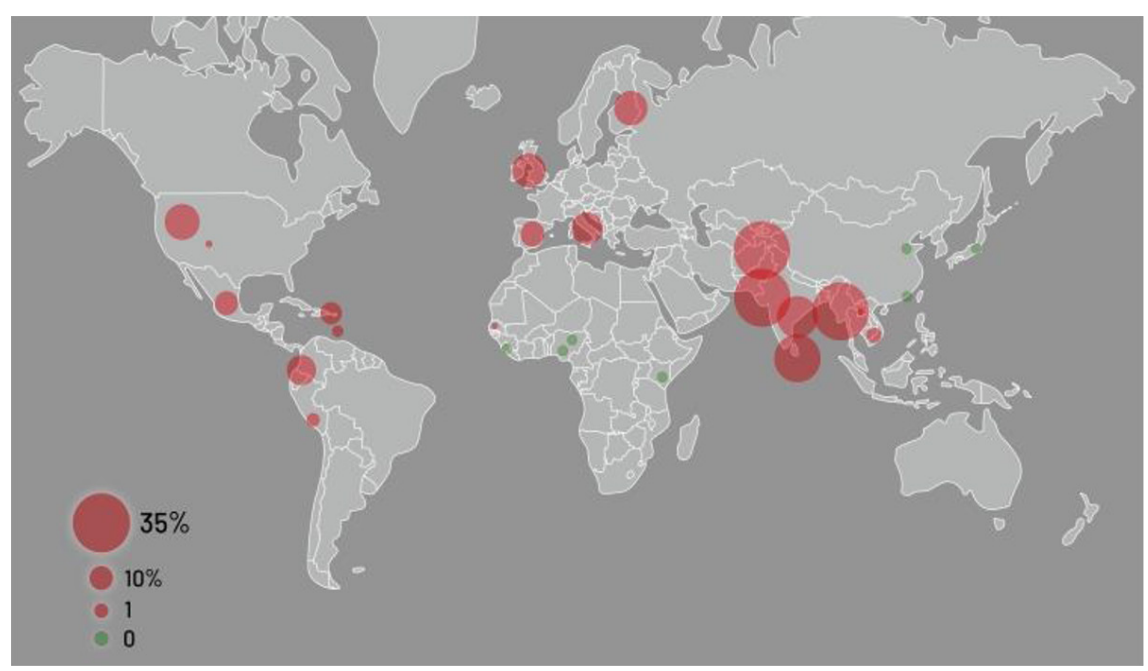

Figure 5: The frequency at which the genetic risk factor is found in various populations around the globe. The geographic distribution of the Neanderthal core haplotype that determines the risk for severe COVID-19 is illustrated in the map.

is inherited from our Neanderthal ancestors can be linked to severe COVID-19 infection and hospitalization.

Neither our genetic background nor environmental factors alone determine our risk of developing severe COVID-19. However, our genes clearly affect both the development and progression of infectious diseases including COVID-19.

Given these considerations, it can be hypothesized that the great differences observed, at least to some extent, can be due to the proportion of Neanderthal genes among the people of these two countries.

\section{Acknowledgment}

The authors would like to thank Mr. Mohammad Reza Hasan-Shahi for producing the excellent figures used in this paper.

\section{Conflict of Interest}

None

\section{References}

1. Romano M, Ruggiero A, Squeglia F, Maga G, Berisio R. A Structural View of SARS-CoV-2 RNA Replication Machinery: RNA Synthe- sis, Proofreading and Final Capping. Cells. 2020;9(5):1267. doi: 10.3390/cells9051267. PubMed PMID: 32443810. PubMed PMCID: PMC7291026.

2. Duffy S. Why are RNA virus mutation rates so damn high? PLOS Biology. 2018;16(8):e3000003. doi: 10.1371/journal. pbio.3000003. PubMed PMID: 30102691. PubMed PMCID: PMC6107253.

3. Mortazavi SAR, Mortazavi SMJ, Sihver L. Selective Pressure-Free Treatments for COVID-19. Radiation. 2020;1(1):18-32. doi: 10.3390/radiation1010003.

4. Koutsakos M, Kedzierska K. A race to determine what drives COVID-19 severity. Nature. 2020;583(7816):366-8. doi: 10.1038/d41586020-01915-3. PubMed PMID: 32661414.

5. Chen M, Wang R, Wang M, Wei GW. Mutations Strengthened SARS-CoV-2 Infectivity. J Mol Biol. 2020;432(19):5212-26. doi: 10.1016/j.jmb.2020.07.009. PubMed PMID: 32710986. PubMed PMCID: PMC7375973.

6. Akst J. Ne anderthal DNA in Modern Human Genomes Is Not Silent. 2020. [cited 2020 October 30]. Available from: https://www. the-scientist.com/features/neanderthal-dna-inmodern-human-genomes-is-not-silent-66299.

7. Huber J. Inherited Neanderthal genes protect us against viruses, study shows. 2018. [cited 
Mortazavi S. A. R. et al

2020 October 5]. Available from: https://scopeblog.stanford.edu/2018/10/05/inherited-neanderthal-genes-protect-us-against-virusesstudy-shows.

8. Zeberg $H$, Pääbo $S$. The major genetic risk factor for severe COVID-19 is inherited from
Neanderthals. Nature. 2020;587:610-2. doi: 10.1038/s41586-020-2818-3.

9. Luo Y. Neanderthal DNA highlights complexity of COVID risk factors. Nature. 2020;587(7835):552-3. doi: 10.1038/d41586020-02957-3. PubMed PMID: 33106620. 